\title{
Septic arthritis in rheumatoid disease causing bilateral shoulder dislocation: diagnosis and treatment assisted by grey scale ultrasonography
}

\author{
B. M. GOMPELS AND L. G. DARLINGTON \\ From the Department of Radiology and Rheumatology Unit, Epsom District Hospital, Epsom, Surrey KTI8 7 EG
}

SUMMARY Signs of sepsis may be obscure in patients with rheumatoid arthritis, particularly in association with long-term steroid therapy. If mortality is to be avoided, a high index of suspicion must be maintained for the diagnosis, and doubtful joints should be aspirated for culture and prompt therapy with the correct antibiotic. In this report a patient with advanced rheumatoid arthritis presented with bilateral shoulder dislocation due to septic arthritis. Ultrasonography was particularly helpful in guiding a $20 \mathrm{G}$ needle to fluid collections with the debris-filled joint capsules and in facilitating successful aspiration. Ultrasound also provided a painless, noninvasive, and safe method of serial assessment of the joints after therapy.

The patient was a 45-year-old ex-nurse with seropositive, erosive, classical rheumatoid arthritis. Before referral to the Epsom Rheumatology Unit she had been treated with penicillamine for 2 years followed in turn by gold, azathioprine, Synacthen, and finally prednisone. At presentation she was receiving $18 \mathrm{mg}$ prednisone daily. She was admitted with 1 week's history of increased joint pain, with malaise and sweating. This acute exacerbation of rheumatoid arthritis with probable septicaemia was treated initially with flucloxicillin and fusidic acid. Penicillin-sensitive Staphylococcus aureus septicaemia was subsequently confirmed on blood culture. No intra-abdominal cause for the septicaemia was detected by ultrasound, and no skin focus was evident.

The patient responded well, but as the septicaemia came under control it was found that shoulder movements had deteriorated significantly. Radiographic and ultrasound examination of her swollen shoulders showed bilateral dislocation by large joint effusions containing calcified debris (Figs 1, 2, and 3 ). With ultrasound control loculated fluid collections within the pyoarthroses were located and aspirated successfully with a $20 \mathrm{G}$ needle. Guidance by ultrasound permitted more complete removal of the sterile pus- $165 \mathrm{ml}$ and $380 \mathrm{ml}$ on the left and right respectively-resulting in a dramatic increase in shoulder movement. This purulent synovial fluid aspirate contained Gram-positive cocci. Sequential examinations with ultrasound were of considerable value in recording the reducing size of the pyoarthroses.

The patient made steady progress, receiving penicillamine, and 10 months later she had an operation on the right shoulder. Copious thin pus was obtained, preventing arthroplasty until culture confirmed its sterility, but 1 month later a Lipmann Kessel shoulder prosthesis was inserted, the glenoid component being screwed into position and the humeral component cemented in place with gentamycin cement (Fig. 4). The clinical result was a reasonable resumption of painless arm movement.

\section{Discussion}

The debilitated patient with rheumatoid arthritis is unusually susceptible to bacterial infection, ${ }^{1}$ particularly in association with Felty's syndrome or high doses of corticosteroids. Kellgren et al. ${ }^{2}$ noted that rheumatoid patients with bacteraemia tended to develop septic arthritis, and the primary source of 


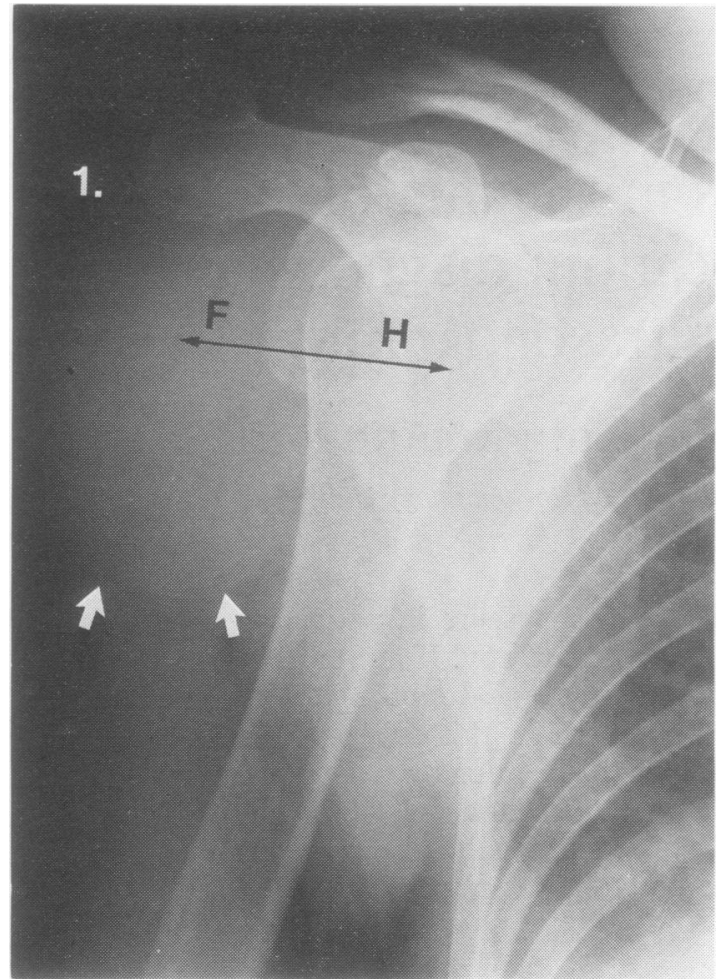

Fig. 1 Radiograph of right shoulder demonstrating dislocation of glenohumeral joint due to large pyoarthrosis. White arrows indicate the pyoarthrosis, which contains calcified debris. Double-headed arrow between $F$ and $H$ represents transverse sectional plane of Fig. 2 and 3. $\mathrm{F}=$ purulent synovial fluid. $\mathrm{H}=$ humerus.

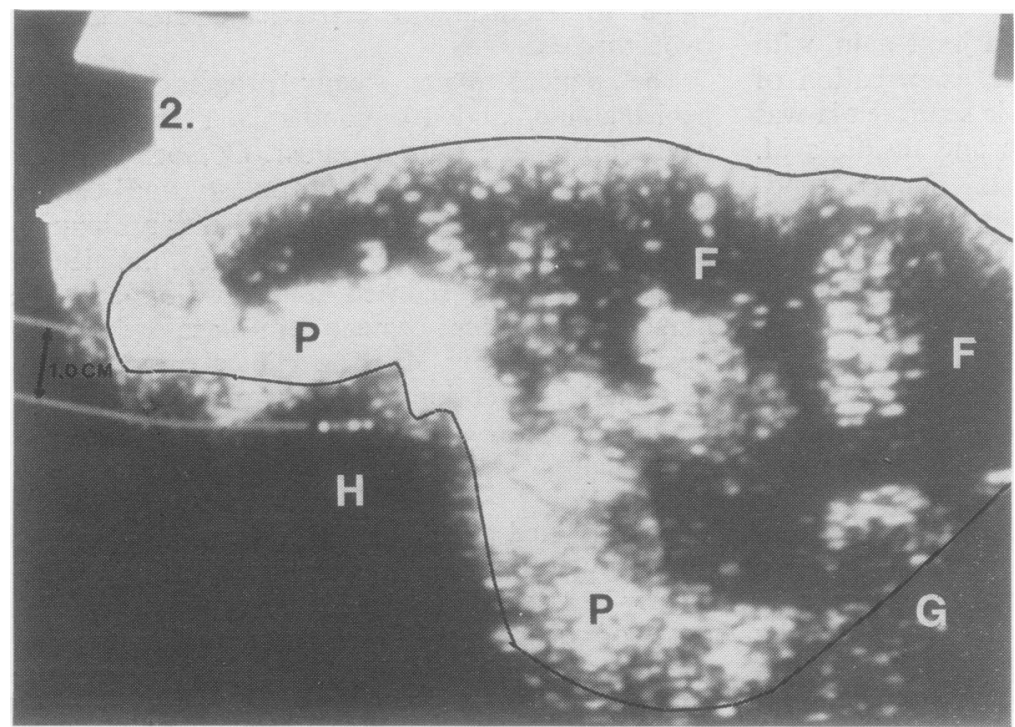

Fig. 2 Transverse ultrasound sectional image along line $\mathbf{F H}$ shown in Fig. 1 . The margins of the distended joint capsule are outlined. A $20 \mathrm{G}$ needle for aspiration of the joint contents was directed towards the echo-free area of purulent synovial fluid $(F)$ within the capsule. The irregular white 'echogenic' areas $(\mathrm{P})$ within this fluid represent the pannus and debris within the joint. $\mathrm{H}=$ humerus. $\mathbf{G}=$ glenoid. 
3.

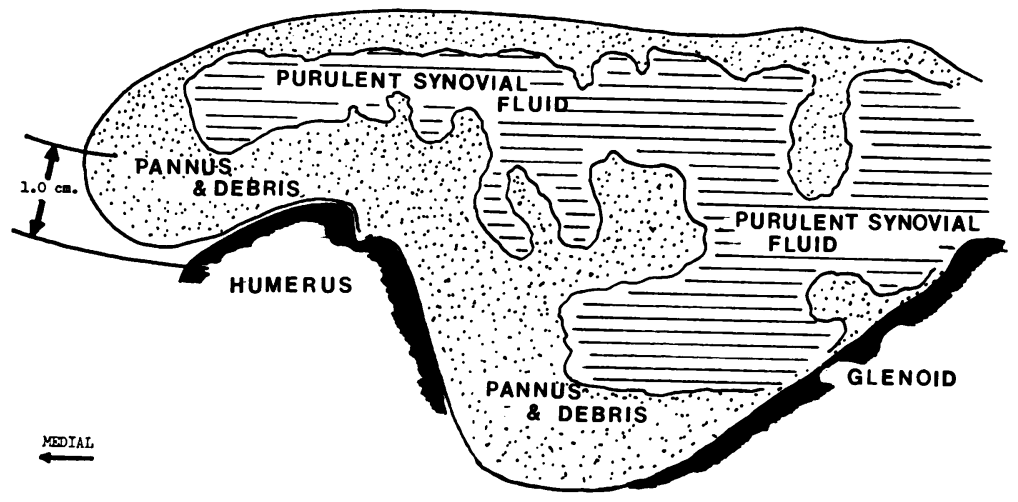

Fig. 3 Diagrammatic representation of Fig. 2.

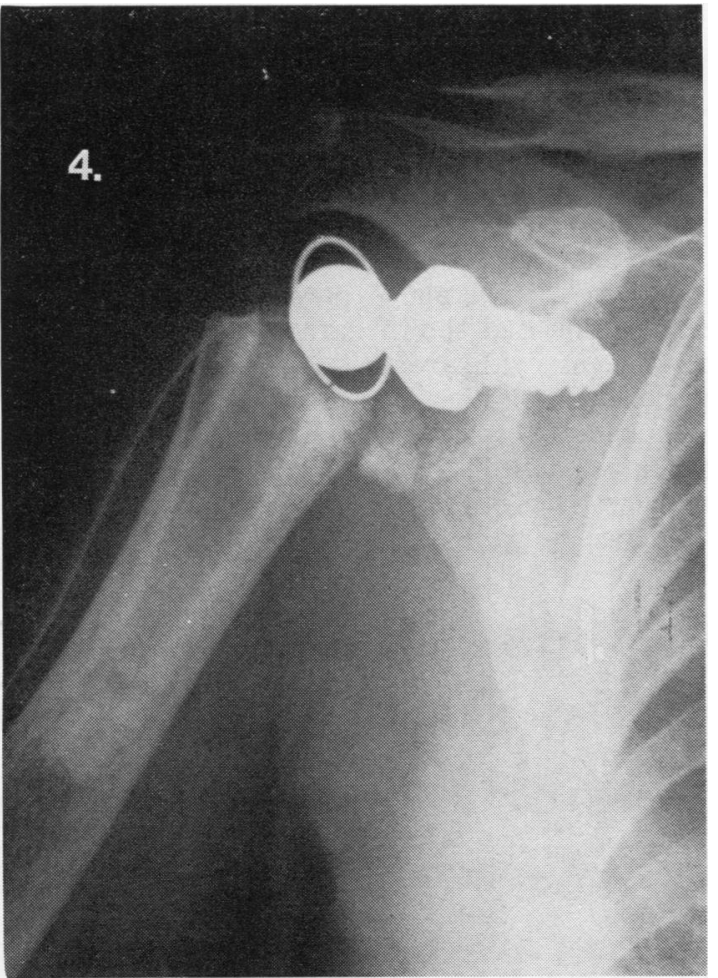

Fig. 4 Radiograph of right shoulder illustrating successful insertion of Lipmann Kessel prosthesis.

infection was frequently proved to be septic skin lesions. ${ }^{3}$ Of the 8 cases of septic arthritis reported by Mitchell et al. ${ }^{4}$ all were due to Staphylococcus aureus, and Newman ${ }^{5}$ has drawn attention to an increasing incidence of penicillin resistance in staphylococci. Mortality may be as high as $42 \%{ }^{2}$ particularly if antibiotic therapy is delayed.
Gristina et al. ${ }^{6}$ recommended arthrotomy, joint irrigation, and systemic antibiotic therapy. Russell and Ansell, ${ }^{7}$ however, used repeated joint aspiration and systemic antibiotics without surgical intervention and obtained excellent results.

Early diagnosis is extremely important. Joint aspiration, in addition to making the patient more comfortable, enables the most suitable antibiotic to be determined from culture, and this must be given in doses sufficient to produce serum and synovial fluid levels reaching the minimum inhibitory concentration for the organism. ${ }^{8}$ Prompt and correct therapy thus limits cartilaginous damage ${ }^{9}$ and minimises the risk of mortality.

\section{References}

1 Hart F D. Complicated rheumatoid disease. $\mathrm{Br} \mathrm{Med} \mathrm{J}$ 1966; ii: $131-5$.

2 Kellgren J H, Ball J, Fairbrother R W, Barnes K L. Suppurative arthritis complicating rheumatoid arthritis. Br Med J 1958; i: 1193-200.

3 Sturrock R D, Cowden E A, Howie E, Greenan D M, Watson-Buchanan $W$. The forgotten nodule: complications of sacral nodules in rheumatoid arthritis. Br Med J 1975; iv: 92-3.

4 Mitchell W S, Brooks P M, Stevenson R D, WatsonBuchanan W. Septic arthritis in ratients with rheumatoid disease: a still underdiagnosed complication. $J$ Rheumatol 1976; 3: 124-33.

5 Newman J H. Review of septic arthritis throughout the antibiotic era. Ann Rheum Dis 1976; 35: 198-205.

6 Gristina A G, Rovere G D, Shoji H. Spontaneous septic arthritis complicating rheumatoid arthritis. J Bone Joint Surg 1974; 56A: $1180-4$.

7 Russell A S, Ansell B M. Septic arthritis. Ann Rheum Dis $1972 ; 31: 40-4$.

8 Cawley M I D, Sattar M A, Barrett S J. Antibiotic concentrations in synovial fluid (S.F.) after oral administration. Presented to the Heberden Society at St Bartholomew`s Hospital June 1980.

9 Curtiss P H Jr, Klein L. Destruction of articular cartilage in septic arthritis. J Bone Joint Surg 1965; 47A: 1595-604. 